\title{
1 Spermidine dampens inflammation by directly 2 inhibiting Th17 cytokine production through a PRDX1 3 associated antioxidant pathway

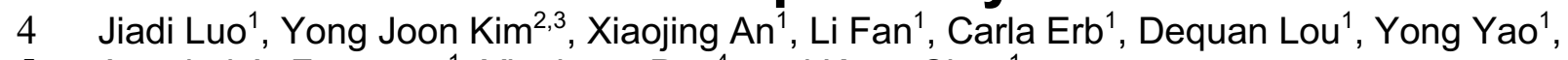 5 Annabel A. Ferguson ${ }^{1}$, Yinghong $\mathrm{Pan}^{4}$, and Kong Chen ${ }^{1}$ \\ ${ }^{1}$ Department of Medicine, University of Pittsburgh, Pittsburgh, PA, United States. ${ }^{2}$ Department of Cell Biology, University of Pittsburgh, Pittsburgh, PA, United States. \\ $9{ }^{3}$ Medical Scientist Training Program, University of Pittsburgh and Carnegie Mellon University, 10 Pittsburgh, PA, United States. \\ $11 \quad{ }^{4}$ PerkinElmer Genomics, Pittsburgh, PA, United States.
}

Running title: Spermidine inhibits IL-17 production

\section{Abstract}

16 The activation of IL-17 signaling has been linked to the pathogenesis of many chronic, inflammatory lung diseases including Cystic Fibrosis (CF). Through unbiased single-cell RNAseq screening, we found that IL-17+ T cells highly express Srm and Smox, which encode two key enzymes for spermidine synthesis. Spermidine has been shown to reduce inflammation by regulating macrophage activation and balancing Th17/Treg differentiation, but its direct effects on Th17 cytokine production has not been carefully investigated. Here, using already differentiated Th17 cells from cultured mouse splenocytes, we found that exogenous spermidine directly inhibits IL-1 $/ /$ L-23 induced IL-17 production. Blockade of endogenous spermidine synthesis enhanced IL-17 production above native levels, further supporting that spermidine is a direct regulator of cytokine secretion independent of differentiation. In vivo, spermidine alleviates lung inflammation in both PA infection and LPS induced acute lung injury models. Further RNA-seq analysis suggests spermidine suppression of Th17 cytokine production is mediated through its PRDX1 dependent antioxidant activity. Our data establishes that spermidine is a direct regulator of Type-17 T cell cytokine production and has potent antiinflammatory effects against lung inflammation. 


\section{Introduction}

34 The Th17/IL-17 signaling pathway is an important regulator of immune and inflammatory

35 function. Th17 cells play a key role in host immunity, particularly in combating infection and

36 maintaining mucosal tissue barrier function. ${ }^{1,2,3,4,5}$ During activation of the Th17/IL-17 pathway,

37 Th17 cells produce IL-17 family cytokines that bind specifically to downstream IL-17 receptors,

38 leading to the release of other inflammatory cell chemokines and stimulating further

39 inflammatory cascades. The Th17/IL-17 pathway thus plays an important immune activating

40 role. Pathologically excessive activation of the Th17/IL-17 response, however, has been

41 implicated in autoimmune and inflammatory disorders such as psoriasis, ankylosing spondylitis,

42 multiple sclerosis, and systemic inflammatory response syndrome (SIRS). ${ }^{1}$

44 Although Th17/IL-17 signaling cascade has been extensively implicated in various physiologic

45 and pathologic contexts, the regulatory mechanisms of the Th17/IL-17 pathway remain unclear.

46 Further delineation of the regulatory mechanism of the Th17/IL-17 signaling pathway is needed

47 to guide clinical management of IL-17 mediated inflammation and autoimmunity.

49 In this study, we unexpectedly found that spermidine synthase (SRM) and spermine oxidase

50 (SMOX) are highly expressed in $/ 117 a+$ cells. SRM and SMOX are the most important catalytic

51 enzymes for the synthesis of spermidine (Spd) from putrescine and spermine, respectively. ${ }^{6,7}$

52 Spermidine is a polyaliphatic amine with anti-inflammatory properties..$^{8,9,10,11,12}$ Recent reports

53 highlighted that spermidine plays important roles in regulating T-cell lineage differentiation. ${ }^{13,14,15}$

54 However, the direct impact of spermidine on Th17 cytokine production has not been carefully

55 examined. In addition, Spd has been linked to a variety of anti-inflammatory mechanisms but

56 has not been directly linked to the regulatory pathway for Th17/IL-17 cytokine secretion prior to

57 this study. 
59 Here we establish that Spd directly regulates the Th17/IL-17 signaling pathway in already

60 differentiated T cells including memory $T$ cells and gamma-delta $T$ cells. The regulatory roles of

61 spermidine and its inhibitors in the Th17/IL-17 pathway were characterized in vitro and in vivo

62 using RNA sequencing, RT-PCR, ELISA, and other technical means. We found that Spd can

63 directly target T cells and downregulate the expression of Th17/IL-17 signaling pathway

64 products, thereby inhibiting IL-17 cytokine-induced inflammation. Conversely, inhibition of Spd

65 synthesis can reverse Spd's suppressive effects on inflammatory cytokine release in the

66 Th17/IL-17 pathway. Further single-cell RNA-seq (scRNA-Seq) results revealed that Spd's

67 regulation of cytokine production depends on the antioxidant activity of peroxiredoxin 1 (PRDX1)

68 in Th17 cells. The results of this study establish Spd's regulatory role in Th17/IL-17 signaling,

69 providing further guidance for the potential pharmacologic management of inflammation and

70 hyper-immunity.

Results

Enzymes for spermidine synthesis (Srm, Smox) are upregulated and Spermidine is

To determine the molecular pathways that may be implicated in Th17/IL-17 signaling, we carefully mined a dataset where mouse peritoneal lavage cells were stimulated with IL-1ß/IL-23 and assessed with scRNA-Seq, with a focus on 4 subsets of T-cell only (Figure 1A).

80 Surprisingly, Srm and Smox genes were found to be upregulated in IL-17+ expressing cells

81 (Figure 1A). Spermidine synthase (Srm) and spermine oxidase (Smox) are both catalytic

82 enzymes for spermidine synthesis. To further explore the relationship between spermidine and

83 Th17/IL-17 pathway, we assessed Srm and Smox expression levels with qPCR in spleen cells

84 and splenic T cells derived from adult mice. Adult mice were used for their matured immune 
85 systems with already differentiated Th17 cells, allowing for the investigation of spermidine's

86 direct role on T cells independent of the differentiation regulatory pathway.

87 RT-PCR analysis confirmed that Srm and Smox are highly expressed in IL-17 producing cells

88 upon stimulation by IL-1ß/IL-23 (Figure 1B).

89 Next, to further explore the link between spermidine and Th17 cytokine production, we directly

90 measured the levels of spermidine and other polyamines (spermine and putrescine) in T cells

91 after Th17/IL-17 pathway activation. Despite the upregulation of spermidine synthesis enzymes,

92 lower spermidine levels were observed after Th17/IL-17 pathway stimulation (Figure 1C)

93 whereas other polyamines remained unchanged (results not shown). The Srm and Smox gene

94 expression profile and changes in spermidine levels in mature T cells suggest that spermidine

95 may be involved in the regulation of the Th17/IL-17 pathway independent of its regulatory role in

96 T cell differentiation.

Spermidine directly targets T cells and down-regulates Th17/IL-17 pathway products in

$100 \quad$ vitro

101

102 To determine the direct effect of spermidine on the Th17/IL-17 pathway, the cytokine expression

103 profile of activated T cells was measured in the presence of exogenous spermidine. We

104 monitored cytokine regulation by measuring mRNA expression (qPCR) and protein levels

105 (ELISA) for Th17 effector cytokines.

106 Upon administration of spermidine, both the gene expression (Figure 2A) and protein levels

107 (Figure 2B) of Th17/IL-17 cytokine products were downregulated in T cells stimulated with IL-

$1081 \beta / I L-23$. We also observed downregulated gene expression and cytokine levels for the Th1

109 effector cytokine, IFN- $y$, which is typically activated in conjunction with the Th17/IL-17 pathway

110 when fighting foreign infections. ${ }^{16,17}$ These results indicate that spermidine acts directly on T 
111 cells in downregulating Th17/IL-17 pathway products. Upon administration of

112 Difluoromethylornithine (DFMO), an inhibitor of spermidine synthesis ${ }^{18}$, the expression of

113 Th17/IL-17 pathway product genes //17a, //17f, I/22 (Figure 2C) and IL-17A protein (Figure 2D)

114 were enhanced above IL-1ß/IL-23 stimulation alone. DFMO did not, however, affect the IFN- $\gamma$

115 pathway (Figure 2C \& 2D). This result reinforces that spermidine may be one of the key native

116 regulatory points specific to the Th17/IL-17 pathway.

118 Spermidine reduces pneumonia progression in LPS induced acute lung injury mouse model by downregulating the Th17/IL-17 pathway

121 With spermidine's role in the negative regulation of Th17/IL-17 signaling established in vitro, we assessed spermidine's effects on Th17/IL-17 signaling using an acute lung injury (ALI) mouse model. The ALI model is based on the context of bacterial pneumonia, where B6 WT mice were

124 inoculated with LPS to induce lung inflammation and injury, with and without coadministration of 125 spermidine. In this acute injury mouse model, spermidine downregulated Th17/IL-17 pathway 126 expression, including //17a, //17f and //22 genes (Figure 3A) and IL-17A cytokine levels (Figure 127 3B). At the same time, the IFN-y pathway, which is closely associated with the Th17/IL-17 128 pathway as previously described, was also inhibited by spermidine with decreased expression 129 of the Ifng gene (Figure $3 A B$ ). Inhibition of native spermidine synthesis with DFMO resulted in 130 the upregulation of Th17/IL-17 pathway genes (I/17a, I/17f, and //22) as well as IL-17A protein 131 expression (Figure 3CD). Consistent with the corresponding in vitro results, DFMO did not affect 132 IFN-y levels in vivo (Figure 3C \& 3D).

133 We assessed the degree of lung inflammation and damage in this mouse model and found that 134 spermidine treated mice had less severe inflammation and lung damage (Figure 3E-G). There 135 was an overall fewer number of immune cells in the BALF of the spermidine treated group 136 (Figure 3E), indicating lower overall inflammation levels. Immune cells from spermidine 
137 administered lungs had higher proportions of nuclear cells and lower proportions of neutrophils

138 (Figure 3F). In addition, edema levels were reduced in spermidine treated groups (Figure 3G).

139 These measurements collectively indicate lower levels of inflammation in the lungs as a result of

140 spermidine treatment. Not only did spermidine result in lower degrees of inflammation, but

141 spermidine also protected against lung damage according to lung histology (Figure 3K).

142 Inhibiting endogenous spermidine synthesis with DFMO promoted lung inflammation and injury,

143 resulting in higher cytotoxicity, proportionally higher neutrophil counts, and elevated levels of

144 lung edema (Figure 3H-J). Elevated levels of injury and inflammatory infiltration in DFMO

145 treated lungs were also evident in lung histology (Figure 3L). Overall, the combination of lower

146 expression of Th17/IL-17 pathway products and protected lung phenotypes suggest that

147 spermidine protects against lung inflammation and injury by downregulating the Th17/IL-17

148 signaling pathway.

Spermidine downregulates the Th17/IL-17 pathway in an acute live bacterial infection

mouse model

153 A live bacterial infection mouse model was used to assess lung bacterial clearance upon

154 spermidine modulation of the Th17/IL-17 pathway. It is well established that the body's

155 response to KP (Klebsiella pneumoniae) and PA (Pseudomonas aeruginosa), two gram-

156 negative bacilli, are mainly mediated by the Th17/IL-17 pathway. ${ }^{19,20}$

157 In a PA infection model, the spermidine treatment group inhibited Th17/IL-17 pathway

158 expression (Figure 4AB) but did not affect PA bacteria clearance (Figure 4C). Spermidine down-

159 regulated Th17/IL-17 pathway expression, including $/ / 17 a, / / 17 f$ and $/ / 22$ genes (Figure 4A) and

160 IL-17A protein expression (Figure 4B). There was no difference in BALF protein concentration

161 between the spermidine treatment group and the control group (results not shown). 
163 Upregulation of Th17/IL-17 pathway expression is expected to enhance bacterial clearance,

164 while inhibiting Th17/IL-17 signaling can lead to bacterial retention. ${ }^{21,22}$ Inhibiting the expression

165 of Th17/IL-17 pathway with spermidine may therefore hinder bacterial clearance in mice, posing

166 a potential concern for elevated infection risk during inflammation management. However, the

167 quantitative measurement of residual bacteria burden in the lungs showed that spermidine did

168 not adversely affect the clearance PA bacteria (Figure 4C). To further investigate this

169 unimpaired bacterial clearance, we directly administered spermidine to different subtypes of KP

170 (KP396, KP-NDM) and PA (PA ATCC10145, PA14) in vitro. Both KP and PA were completely

171 eradicated after 4 hours of spermidine treatment at spermidine concentrations of $\geq 2 \mathrm{mM}$ (Figure

172 4D). The bactericidal effect of spermidine after $24 \mathrm{~h}$ of treatment is the same as that of after $4 \mathrm{~h}$

173 (Figure 4E). Direct bactericidal effect of spermidine may aid in bacterial clearance, maintaining

174 mucosal barrier homeostasis despite the downregulation of Th17/IL-17 pathway expression.

176 To further determine whether spermidine targets $T$ cells for reducing inflammation, we used $T$

177 cell knockout mice to explore whether spermidine acts on other immune cells. We established

178 LPS-induced acute lung injury and live bacterial infection models in Rag2/II2rg double gene

179 knockout mice, which are deficient in T cells, B cells and NK cells. ${ }^{24}$ The Th17 cytokines were

180 completely absent in these mouse according to RT-PCR (data not shown), confirming that T

181 cell function were knocked out. According to both ALI (Figure 5A-C) and PA14 infection (Figure

182 5D-F) models, spermidine did not protect against inflammation in Rag2/II2rg double gene

183 knockout mice. All markers of inflammation were elevated with or without spermidine

184 administration, including the number of BAL immune cells, the proportion of BAL monocytes, the

185 proportion of BAL neutrophils, BAL total protein, wet-to-dry specific gravity, edema, and lung

186 histopathological score (Figure 5). This stark loss in spermidine's anti-inflammatory effect

187 accentuates that spermidine is likely acting upon Th17 T cells in the Th17/IL-17 pathway and

188 not through other immune cells. 
190 Spermidine down-regulates Th17/IL-17 cytokine gene expression by regulating PRDX1

191 activity

192

193 To determine the intrinsic mechanism of how effector Th17 cytokines are downregulated by

194 spermidine in T cells, we performed scRNA-Seq sequencing and bulk mRNA sequencing

195 analysis on IL-1ß/IL-23 activated splenic T cells (SPT) and lymph node (LN) cells treated with

196 spermidine. To minimize batch effect between samples, we multiplex all the samples with cell

197 hashing method ${ }^{23}$ for spermidine treatment (SPT sper and LN sper) and untreated groups (SPT

198 stim and LN stim) (Figure 6A). The results after de-multiplexing ${ }^{24}$ show that, compared with the

199 spermidine untreated T cell control group (SPT stim), the expression of Txn1 and Prdx1 genes

200 were significantly increased in the spermidine treatment group (Figure 6B-C). Prdx1 encodes

201 peroxidase 1 (PRDX1), which is an antioxidant enzyme that protects the cell by reducing

202 hydrogen peroxide retention. ${ }^{25,26}$ Coincidentally, thioredoxin (TXN1) is an oxidoreductase also

203 with antioxidant activities ${ }^{27,28}$. Other genes such as Txnrd1 and Stat1 did not change

204 significantly. We further confirmed with mRNA-Seq data that the expression of Txn1 and Prdx1

205 genes in T cells in the spermidine treatment group was increased directly in proportion to the

206 concentration of spermidine (Figure 5D). In the subsequent RT-PCR verification, we observed

207 that only the Prdx1 gene was highly expressed in the spermidine treatment group (Figure 5E).

208 Prdx1 activity has an especially strong association with spermidine treatment, raising the

209 possibility of Prdx1 being a key spermidine effector.

210 To determine whether spermidine regulates Th17 cytokine production through PRDX1,

211 adenanthin was co-administered with spermidine into activated Th17 cells. Adenanthin is a

212 recently discovered inhibitor against antioxidant enzymes, including PRDX1, derived from a

213 plant extract ${ }^{29,30}$. As previously observed, IL-1ß/IL-23 activated the Th17/IL-17 and IFN-Y

214 pathways, while spermidine significantly down-regulated Th17/IL-17 and IFN-y products (Figure 
215 5F). Compared to spermidine treatment alone, the addition of adenanthin enhanced the gene

216 expression of $/ / 17 a, I / 17 f$, I/22 as well as the levels of Th17/IL-17 products (Figure $5 \mathrm{~F}-\mathrm{G}$ ).

217 Adenanthin successfully reversed the inhibitory effect of spermidine on Th17/IL-17 pathway,

218 suggesting that spermidine down-regulates Th17/IL-17 pathway by regulating PRDX1 activity.

219 Interestingly, the combination of adenanthin and spermidine treatment further inhibited the gene

220 and protein expression of IFN-y (Figure 5F-G), which needs to be further explored in future

221 investigations.

223 Adenanthin and DFMO are both inhibitors against spermidine's action against Th17/IL-17

224 signaling. DFMO inhibits upstream of spermidine action during spermidine synthesis, while 225 adenanthin inhibits downstream of spermidine by inhibiting PDRX1. Administration of DFMO 226 reverses the inhibitory effect of spermidine on the Th17/IL-17 pathway and enhances the 227 expression of Th17/IL-17 pathway products. In contrast, coadminstration of adenanthin with 228 spermidine did not completely restore the expression of Th17/IL-17 pathway products. This 229 suggests that the spermidine's activity is not completely inhibited by suppressing PDRX1 230 activity. These results indicate that spermidine may inhibit the expression of Th17/IL-17

231 products through additional mechanisms, which need to be investigated further.

235 The key position of Th17/IL-17 signaling pathway in the body's immune system highlights the

236 importance of studying the regulatory mechanism of this pathway. Understanding the regulation

237 mechanisms of the Th17/IL-17 signaling pathway, especially the regulatory factors of Th17

238 cells, have always been of great interest for immunology research. Th17 cells have been

239 reported to be inhibited by cytokines secreted by most other types of T cells such as Th1 cells

240 and Th2 cells, ${ }^{31,32,33}$ which have important roles in controlling inflammatory cascades and 
241 hyper-immunity. Regulatory T cells play a role in monitoring and regulating Th17 cells, but the

242 specific regulatory mechanisms for the Th17/IL-17 signaling pathway has been elusive. ${ }^{34,35}$

244 Polyamines, especially spermidine and spermine, have been implicated with inflammation, with 245 elevated levels of polyamines found in the inflammatory sites for infection, trauma, tumor and 246 autoimmune diseases. ${ }^{36,37}$ Prior to this study, the mechanism through which polyamines enact 247 their anti-inflammatory effects had not yet been established. In this study, we established a key 248 regulatory mechanism of spermidine on Th17/IL-17 signaling pathway. Based on both in vitro 249 and in vivo models, we found that spermidine directly acts on T cells to down-regulate the 250 Th17/IL-17 signaling pathway. Based on RNA sequencing data, we determined that spermidine 251 down-regulates the Th17/IL-17 signaling pathway by controlling PRDX1 protein activity in T 252 cells. PRDX1 protein is mainly involved in antioxidant functions and helps to remove reactive 253 oxygen species (ROS) in cells. PRDX1 activity is important during times of cellular oxidative 254 stress, such as in inflammation, where excessive amounts of reactive oxygen species are 255 produced in the cell. ${ }^{38,39}$ Our observation that spermidine acts through PRDX1 is consistent with 256 the literature that spermidine is involved in controlling oxidative stress. ${ }^{40,41,42}$

258 We also found that spermidine treatment, despite its immune suppressive role, does not 259 negatively affect lung bacterial clearance in mice. In addition to its anti-inflammatory and 260 immunomodulatory effects, spermidine was found to have a direct bactericidal effect against PA 261 and KP. These results suggest that spermidine may be key factor in the host-infection interface, 262 maintaining mucosal barrier homeostasis by controlling infection without detrimental levels of 263 inflammation. Further understanding of how spermidine endogenously controls the dynamic 264 balance between Th17/IL-17 suppression and infection will guide clinical strategies against 265 infection induced hyper-immunity. 
267 Further investigations that explore other molecular mechanisms by which spermidine inhibits the

268 Th17/IL-17 are necessary. Our comparison of DFMO and adenanthin, inhibitors of spermidine

269 synthesis and effectors, respectively, indicate that spermidine may have additional Th17/IL-17

270 pathway effectors besides PRDX1. In addition, whether other polyamines, which also have been

271 implicated with anti-inflammatory effects, ${ }^{36,37}$ engage in a similar regulatory mechanism as

272 spermidine must also be investigated. Furthering the mechanistic understanding of Th17/IL-17

273 regulation by endogenous and exogenous spermidine may aid efforts to clinically manage

274 debilitating cases of infection and autoimmune diseases.

275

276 Materials and methods

277 1. Mouse models. All the mice used in this project were of wildtype strains and purchased from

278 Jackson Lab (Cat\# 000664). Animals were maintained in pathogen-free conditions at the core

279 animal facility at the University of Pittsburgh Medical Center. Mice age matched to 6-8 weeks

280 old were used to set up pre-designed models with the approval from the University of Pittsburgh

281 Institutional Animal Care and Use Committee.

2821.1 Mouse peritoneal neutrophil induction and treatment. 4\% sterile Thioglycollate (Sigma, Cat\#

283 70157) in $\mathrm{ddH}_{2} \mathrm{O}$ was applied to induce mice peritonitis, $100 \mu$ per mouse with intraperitoneal

284 (IP) injection. $5 \mathrm{~h}$ later, mice were sacrificed by $\mathrm{CO}_{2}$ euthanasia. Peritoneal lavage samples

285 were collected by injecting sterile PBS into peritoneal cavity with a $10 \mathrm{ml}$ syringe. Peritoneal

286 lavage cells were isolated and stimulated overnight at $37^{\circ} \mathrm{C}$ with $50 \mathrm{ng} / \mathrm{ml} \mathrm{m}$-IL-1 $\beta$ (Biolegend,

287 Cat\# 575002) plus m-IL-23 (Biolegend, Cat\# 589002) in 10\% FBS-IMDM media. Cells were

288 collected for scRNA-seq analysis.

2891.2 Mouse lung inflammation induction and treatment. Either LPS (Sigma Inc.) or P.A. (ATCC®

290 10145GFP TM) were inoculated into mice to mediate acute mouse lung inflammation. Spermidine

291 (Sigma, Cat\# S2626-1G) or the spermidine synthesis inhibitor DL-a-Difluoromethylornithine 
292 (DFMO, Cayman Chemical, Cat\# 16889) were administered at time of inoculation to explore the

293 roles of spermidine in inflammation regulation. Three modules were designed in this project:

294 LPS administration (5 $\mu \mathrm{g} / \mathrm{g}$ body weight) + spermidine treatment ( $5 \mu \mathrm{g} / \mathrm{g}$ body weight); P.A.

295 infection $\left(1 \times 10^{6} \mathrm{cfu} /\right.$ mouse $)+$ spermidine treatment $(5 \mu \mathrm{g} / \mathrm{g}$ body weight); LPS administration (5

$296 \mu \mathrm{g} / \mathrm{g}$ body weight $)+$ DFMO treatment $(1.5 \mathrm{mg} / \mathrm{g}$ body weight $)$. For spermidine treatment

297 modules, mice were injected intraperitoneally with spermidine solution or PBS as control, then

298 anesthetized with isoflurane and challenged with LPS or PA intratracheally (IT). For DFMO

299 treatment experiments, mice were pretreated with DFMO or PBS intraperitoneally for $1 \mathrm{hr}$, after

300 which all mice were anesthetized with isoflurane and intratracheally inoculated with LPS. 18h

301 after inoculation, mice from all the modules were euthanized with $\mathrm{CO}_{2}$ for further processing.

302 2. BAL and lung tissue processing. Before harvesting the lungs, mouse lungs were lavaged with

303 sterile PBS through the trachea. BAL cells were obtained by centrifuging the BAL fluid at $300 \times \mathrm{xg}$

304 for $10 \mathrm{~min}$ at $4{ }^{\circ} \mathrm{C}$. Right after centrifuging from the cytocentrifuge (Cytospin 4 , Thermo

305 Scientific), fresh BAL cell cytospin slides were stained using a Kwik-Dif Stains kit (Fisher

306 Scientific, \#99-907-00) for inflammatory cell differential counts. Right superior and inferior lobes

307 were homogenized in $1 \mathrm{ml}$ sterile PBS using gentleMACS Octo Dissociator (Miltenyi Biotec). A

$308100 \mu \mathrm{l}$ aliquot of homogenate was reserved for bacteria cfu assay. The rest of the homogenate

309 was centrifuged at $4{ }^{\circ} \mathrm{C}$ over $10000 \mathrm{~g}$ for $10 \mathrm{~min}$, and the supernatant was stored at $-80{ }^{\circ} \mathrm{C}$ for

310 further use in ELISA, LDH assays, and other experiments. The right middle lobe was cut down

311 and incubated in a $65^{\circ} \mathrm{C}$ incubator for $48 \mathrm{~h}$, and the wet/dry weight ratio as well as the edema

312 levels were measured. The left inferior lobe was immersed in RLT buffer with 2-

313 mercaptoethanol for RNA extraction. The left superior lobe was fixed with $10 \%$ neutral buffered

314 formalin (Sigma, Cat\# HT501128-4L) at $4{ }^{\circ} \mathrm{C}$ for over $24 \mathrm{~h}$ and subsequently subjected to H\&E

315 staining. 
316 3. LDH detection. LDH Cytotoxicity Assay (Promega, Cat\# G1780) was used to quantify LDH

317 levels as a function of cytotoxicity. The procedure was strictly performed according to the

318 manufacturer's protocol.

319 4. Bactericidal experiments by spermidine. Live bacteria including KP396, KP-NDM, PA and

320 PA14 were treated with 0, 0.5, 1, 2, 3 and 4mM spermidine for $4 \mathrm{~h}$ and $24 \mathrm{~h}$ respectively. After

321 the given time points, bactericidal activity was determined by using a CFU assay.

322 5. Bacteria CFU assay. This assay was used to accurately assess bacteria burden. Briefly, the

323 primary sample was serially diluted as 1:10, 1:100, 1:1000, 1:10000, 1:100000, 1:1000000. A10

$324 \mu$ aspirate from each diluted sample were transferred to a LB agar plate in technical triplicates.

325 The agar plates were incubated at $37^{\circ} \mathrm{C}$ overnight and the resulting bacteria colonies were

326 counted carefully. The final CFU calculation formula was as followed: the bacterial concentration

327 in the sample tested $(\mathrm{CFU} / \mathrm{ml})=($ Total number of colonies from the triplicate dots $/ 3)$ *dilution

328 factor*100.

329 6. Primary T cell isolation. Fresh T cells from spleen and lung draining lymph node were used

330 for in vitro experiments. Spleen and lung draining lymph nodes were harvested from WT mice

331 after $\mathrm{CO}_{2}$ euthanasia. Single cell suspensions from spleen and lymph node tissue were

332 collected separately by crushing the organs with sterile, chilled PBS through a $70 \mu \mathrm{m}$ filter

333 (Fisher Scientific, Cat\# 22-363-548) and a syringe.

334 A mouse T cell isolation kit (STEMCELL, Cat\# 19851) was used to enrich T cells from harvested

335 spleen cells. Unwanted cells were negatively purified from spleen cell suspensions by

336 incubating spleen cells with biotinylated antibodies directed against non-T cells. The unwanted

337 cells were magnetically isolated from total spleen cells with a streptavidin-coated magnetic

338 bead, enriching the spleen cell suspension with T cells. 
7. Primary cell stimulation. Enriched spleen T cells and lung draining lymph node cells were plated $\left(3-4 \times 10^{6}\right.$ cells) in a 24-well plate, using $0.5 \mathrm{ml}$ IMEM, 10\% FBS, and standard Penicillin-

341 Streptomycin antibiotics in each well. Cells were treated with various interventional conditions,

342 as followed: $50 \mathrm{ng} / \mathrm{ml} \mathrm{m}-\mathrm{IL}-1 \beta / \mathrm{m}-\mathrm{IL}-23 ; 50 \mathrm{ng} / \mathrm{ml} \mathrm{m}-\mathrm{IL}-1 \beta / \mathrm{m}-\mathrm{IL}-23+3 \mu \mathrm{M}$ spermidine; $50 \mathrm{ng} / \mathrm{ml}$

$343 \mathrm{~m}-\mathrm{IL}-1 \beta / \mathrm{m}-\mathrm{IL}-23+20 \mathrm{mM}$ DFMO; $50 \mathrm{ng} / \mathrm{ml} \mathrm{m}$-IL-1ß/m-IL-23+3 $\mu \mathrm{M}$ spermidine+10 $\mu \mathrm{M}$ Ade

344 (ChemFaces, Cat\# CFN99215). For DFMO experiments, DFMO and control PBS were added to

345 the cells $4 \mathrm{~h}$ prior to IL-1 $\beta / \mathrm{IL}-23$ stimulation to block spermidine synthesis. For other

346 experimental groups, all treatments were administered to the cells at the time of stimulation. At

$34718 \mathrm{~h}$ time point, cells were harvested for RNA-seq, spermidine quantification by HPLC, or lysed

348 in RLT buffer with 2-mercaptoethanol for gene expression. The supernatant was used for ELISA

349 experiments.

350 8. RNA Extraction and cDNA Synthesis. Cell and lung tissue RNA was extracted with RNeasy

351 Miniprep Kit (QIAGEN, Cat\# 74136), in accordance with the manufacturer's instruction. cDNA

352 was synthesized using qScriptTM cDNA Synthesis Kits (Quantabio, Cat\# 95047-100).

353 9. Real-Time PCR. Real-time PCR was constructed and read by the Bio-Rad CFX96 system

354 using TaqMan PCR Master Mix (Bio-Rad, Cat\# 1725284) and pre-mixed probe sets: //17a

355 (Mm00439618_m1), II17f(Mm00521423_m1), I/22 (Mm01226722_g1), Ifng

356 (Mm01168134_m1), Srm (Mm00726089_s1), Smox (Mm01275475_m1), Prdx1

357 (Mm01621996_s1), Gapdh (Mm99999915_g1) and Hprt (Mm03024075_m1), from Thermo

358 Scientific.

359 10. ELISA. ELISA kits were used to measure mouse IL-17A (BioLegend, Cat\# 432506) and

360 mouse IFN-ץ (BioLegend, Cat\# 430804). The procedures were strictly employed according to 361 the manufacturer's instructions. 
11. ScRNA-seq, RNA-seq and Total-seq. ScRNA-seq libraries were constructed and sequenced following the "Single Cell 3' Reagent Kits v2 User Guide" (10X Genomics). RNA-seq

364 methodology was developed in close reference to a published study. ${ }^{43}$ Similar to ScRNA-seq,

365 Total-seq was performed following the cell hashing protocol (BioLegend). Briefly, five groups of

366 cells were pre-labelled with mouse HashTag oligos (HTO) on cell surface: spleen T cell control

367 group (SPT ctrl) with hashtag 1 (BioLegend, Cat\# 155801); spleen T cell IL-1ß/IL-23 stimulation

368 group (SPT stim) with hashtag 2 (BioLegend, Cat\# 155803); spleen T cell IL-1ß/IL-23 plus

369 spermidine treatment group (SPT sper) with hashtag 3 (BioLegend, Cat\# 155805); lymph node

370 cell IL-1ß/IL-23 stimulation group (LN stim) with hashtag 4 (BioLegend, Cat\# 155807); Iymph

371 node cell IL-1ß/IL-23 plus spermidine treatment group (LN sper) with hashtag 5 (BioLegend,

372 Cat\# 155809). The five cell populations were mixed and barcoded, and barcoded cDNA were

373 synthesized via reverse transcription. Further cDNA libraries were constructed by cDNA

374 amplification. HTO-derived cDNAs (<180bp) and mRNA-derived cDNAs (>300bp) were

375 separated using a magnetic beads selection methodology. An HTO sequencing library was

376 generated for sequencing with another round of cDNA amplification.

377 mRNA-derived cDNA library was constructed following the conventional scRNA-seq protocol.

378 HTO library and DNA library were then sequenced on an Illumina HiSeq sequencer

379 (MedGenome, Inc.). Sequencing data was initially analyzed by Cell Ranger (10X Genomics)

380 and downstream analysis was done by $\mathrm{R}$.

381 12. Statistics. All data analyses were achieved and graphed by Prism 8.0 (GraphPad). The one-

382 way ANOVA test was performed to compare differential gene expression among four groups.

383 Paired Student's t-test was applied for other comparisons between the paired two groups,

\section{Data Availability}

385 R code used for RNA-seq analysis is available upon request. The sequencing data reported in 386 this paper will be deposited to the Gene Expression Omnibus. 
bioRxiv preprint doi: https://doi.org/10.1101/2021.08.31.458409; this version posted August 31, 2021. The copyright holder for this preprint (which was not certified by peer review) is the author/funder. All rights reserved. No reuse allowed without permission.

387 
References:

389

1. Pappu, R., Ramirez-Carrozzi, V. \& Sambandam, A. The interleukin-17 cytokine family: critical players in host defence and inflammatory diseases. Immunology 134, 8-16 (2011).

2. Dubin, P.J. et al. Interleukin-23-mediated inflammation in Pseudomonas aeruginosa pulmonary infection. Infect Immun 80, 398-409 (2012).

3. Beisswenger, C. et al. Moxifloxacin modulates inflammation during murine pneumonia. Respir Res 15, 82 (2014).

4. $\mathrm{Xu}, \mathrm{X}$. et al. Role of Interleukin-17 in defense against pseudomonas aeruginosa infection in lungs. Int J Clin Exp Med 7, 809-816 (2014).

5. Dubin, P.J. \& Kolls, J.K. IL-23 mediates inflammatory responses to mucoid Pseudomonas aeruginosa lung infection in mice. Am J Physiol Lung Cell Mol Physiol 292, L519-528 (2007).

6. Pegg, A.E. Mammalian polyamine metabolism and function. IUBMB Life 61, 880-894 (2009).

7. Pegg, A.E. The function of spermine. IUBMB Life 66, 8-18 (2014).

8. Moron, B. et al. Activation of protein tyrosine phosphatase non-receptor type 2 by spermidine exerts anti-inflammatory effects in human THP-1 monocytes and in a mouse model of acute colitis. PLoS One 8, e73703 (2013).

9. Choi, Y.H. \& Park, H.Y. Anti-inflammatory effects of spermidine in lipopolysaccharidestimulated BV2 microglial cells. J Biomed Sci 19, 31 (2012).

10. Jeong, J.W. et al. Spermidine Protects against Oxidative Stress in Inflammation Models Using Macrophages and Zebrafish. Biomol Ther (Seoul) 26, 146-156 (2018).

11. Yang, Q. et al. Spermidine alleviates experimental autoimmune encephalomyelitis through inducing inhibitory macrophages. Cell Death Differ 23, 1850-1861 (2016).

12. Paul, S. \& Kang, S.C. Natural polyamine inhibits mouse skin inflammation and macrophage activation. Inflamm Res 62, 681-688 (2013).

13. Carriche, Guilhermina M., et al. "Regulating T-cell differentiation through the polyamine spermidine." Journal of Allergy and Clinical Immunology 147.1 (2021): 335-348.

14. Puleston, Daniel J., et al. "Polyamine metabolism is a central determinant of helper T cell lineage fidelity." Cell (2021). 
15. Wagner, Allon, et al. "Metabolic modeling of single Th17 cells reveals regulators of autoimmunity." Cell (2021).

16. Shi, Yun, et al. "Helicobacter pylori-induced Th17 responses modulate Th1 cell responses, benefit bacterial growth, and contribute to pathology in mice." The Journal of Immunology184.9 (2010): 5121-5129.

17. Wang, C. F., et al. "Prominent contribution of Th1, Th17, and Tregs to the host response during M. neoaurum infection." Genet Mol Res 15.3 (2016).

18. Gamble, L.D. et al. Inhibition of polyamine synthesis and uptake reduces tumor progression and prolongs survival in mouse models of neuroblastoma. Sci Transl Med $\mathbf{1 1}$ (2019).

19. Chen, K. et al. IL-17 Receptor Signaling in the Lung Epithelium Is Required for Mucosal Chemokine Gradients and Pulmonary Host Defense against K. pneumoniae. Cell Host Microbe 20, 596-605 (2016).

20. Bayes, H.K., Ritchie, N.D. \& Evans, T.J. Interleukin-17 Is Required for Control of Chronic Lung Infection Caused by Pseudomonas aeruginosa. Infect Immun 84, 3507 3516 (2016).

21. Ye, P. et al. Interleukin-17 and lung host defense against Klebsiella pneumoniae infection. Am J Respir Cell Mol Biol 25, 335-340, doi:10.1165/ajrcmb.25.3.4424 (2001). 15.

22. Ye, P. et al. Requirement of interleukin 17 receptor signaling for lung CXC chemokine and granulocyte colony-stimulating factor expression, neutrophil recruitment, and host defense. J Exp Med 194, 519-527, doi:10.1084/jem.194.4.519 (2001).

23. Stoeckius, Marlon, et al. "Cell Hashing with barcoded antibodies enables multiplexing and doublet detection for single cell genomics." Genome biology 19.1 (2018): 1-12.

24. Xin, Hongyi, et al. "GMM-Demux: sample demultiplexing, multiplet detection, experiment planning, and novel cell-type verification in single cell sequencing." Genome biology 21.1 (2020): 1-35.

25. Wu, C. et al. Sulfonation of the resolving cysteine in human peroxiredoxin 1: A comprehensive analysis by mass spectrometry. Free Radic Biol Med 108, 785-792 (2017).

26. El Eter, E. \& Al-Masri, A.A. Peroxiredoxin isoforms are associated with cardiovascular risk factors in type 2 diabetes mellitus. Braz J Med Biol Res 48, 465-469 (2015).

27. Nordberg, J. \& Arner, E.S. Reactive oxygen species, antioxidants, and the mammalian thioredoxin system. Free Radic Biol Med 31, 1287-1312 (2001). 
28. Mohammadi, F., Soltani, A., Ghahremanloo, A., Javid, H. \& Hashemy, S.I. The thioredoxin system and cancer therapy: a review. Cancer Chemother Pharmacol 84, 925 935 (2019).

29. Siernicka, M. et al. Adenanthin, a new inhibitor of thiol-dependent antioxidant enzymes, impairs the effector functions of human natural killer cells. Immunology 146, 173-183

30. Liu, C.X. et al. Adenanthin targets peroxiredoxin I and II to induce differentiation of leukemic cells. Nat Chem Biol 8, 486-493 (2012).

33. Harrington, L.E. et al. Interleukin 17-producing CD4+ effector T cells develop via a lineage distinct from the $\mathrm{T}$ helper type 1 and 2 lineages. Nat Immunol 6, 1123-1132 (2005).

34. Ichiyama, K. et al. Foxp3 inhibits RORgammat-mediated IL-17A mRNA transcription through direct interaction with RORgammat. J Biol Chem 283, 17003-17008 (2008).

35. Zhou, L. et al. TGF- $\beta$-induced Foxp3 inhibits $\mathrm{T}(\mathrm{H}) 17$ cell differentiation by antagonizing RORgammat function. Nature 453, 236-240 (2008).

36. Merentie, M. et al. Oxidative stress and inflammation in the pathogenesis of activated polyamine catabolism-induced acute pancreatitis. Amino Acids 33, 323-330 (2007).

37. Thomas, T.J., Gunnia, U.B., Seibold, J.R. \& Thomas, T. Defective signal-transduction pathways in T-cells from autoimmune MRL-lpr/lpr mice are associated with increased polyamine concentrations. Biochem J 311 ( Pt 1), 175-182 (1995).

38. Brune, B. et al. Redox control of inflammation in macrophages. Antioxid Redox Signal 19, 595-637 (2013).

39. Mills, E.L. \& O'Neill, L.A. Reprogramming mitochondrial metabolism in macrophages as an anti-inflammatory signal. Eur J Immunol 46, 13-21 (2016).

40. Tkachenko, A.G., Akhova, A.V., Shumkov, M.S. \& Nesterova, L.Y. Polyamines reduce oxidative stress in Escherichia coli cells exposed to bactericidal antibiotics. Res Microbiol 163, 83-91 (2012). 
525
41. Chattopadhyay, M.K., Tabor, C.W. \& Tabor, H. Polyamines protect Escherichia coli cells from the toxic effect of oxygen. Proc Natl Acad Sci U S A 100, 2261-2265 (2003).

42. Tkachenko, A.G. \& Fedotova, M.V. Dependence of protective functions of Escherichia coli polyamines on strength of stress caused by superoxide radicals. Biochemistry (Mosc) 72, 109-116 (2007).

43. Ray, Mondira, et al. "RNA-seq in pulmonary medicine: how much is enough?." American journal of respiratory and critical care medicine 192.3 (2015): 389391. 


\section{Spermidine dampens inflammation through inhibiting Th17 cytokine production}

\section{Spermidine inhibits IL-17 production}

Jiadi Luo ${ }^{1}$, Yong Joon Kim ${ }^{2,3}$, Xiaojing An ${ }^{1}$, Li Fan ${ }^{1}$, Carla Erb ${ }^{1}$, Dequan Lou ${ }^{1}$, Yong Yao ${ }^{1}$, Annabel A. Ferguson ${ }^{1}$, Yinghong Pan $^{4}$, and Kong Chen ${ }^{1}$

${ }^{1}$ Department of Medicine, University of Pittsburgh, Pittsburgh, PA, United States. ${ }^{2}$ Department of Cell Biology, University of Pittsburgh, Pittsburgh, PA, United States.

${ }^{3}$ Medical Scientist Training Program, University of Pittsburgh and Carnegie Mellon University, Pittsburgh, PA, United States.

${ }^{4}$ PerkinElmer Genomics, Pittsburgh, PA, United States. 
bioRxiv preprint doi: https://doi.org/10.1101/2021.08.31.458409; this version posted August 31, 2021. The copyright holder for this preprint A Ccr7 (which was not certified by peer review) is the author/funder. All rights reserved. No reuse allowe

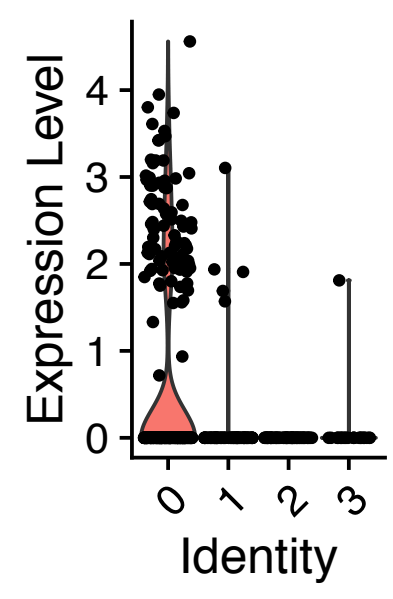

II17a

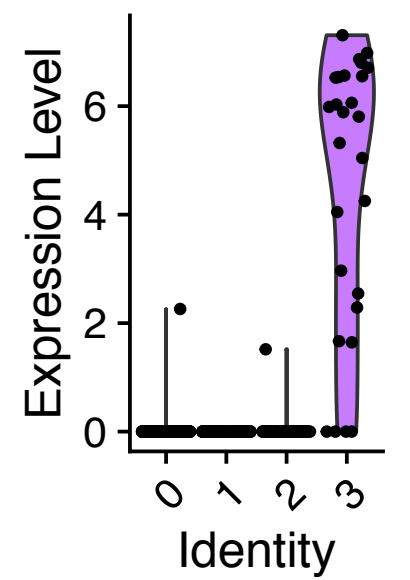

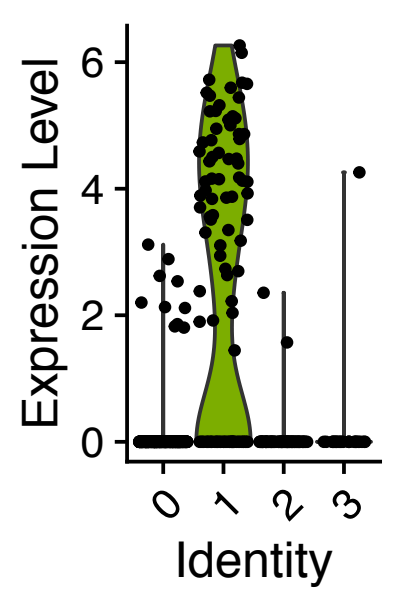

Srm

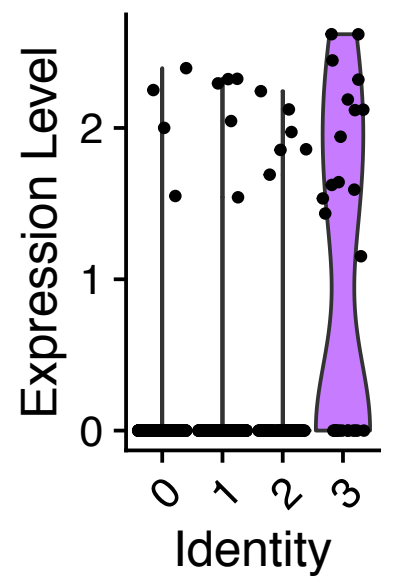

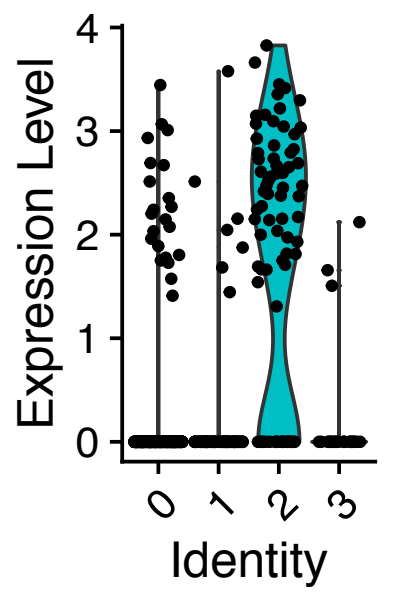

Smox

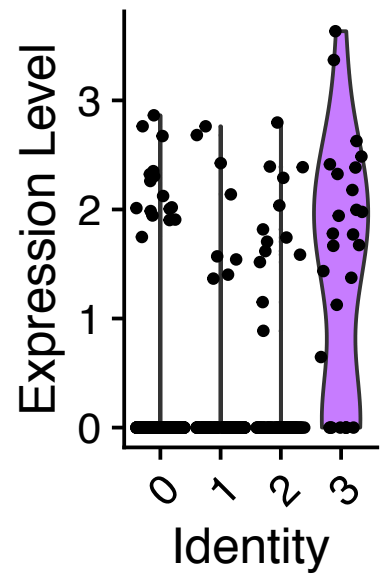

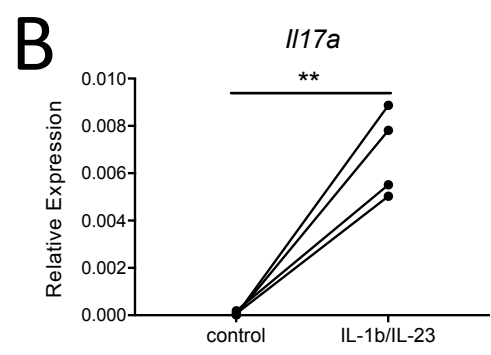
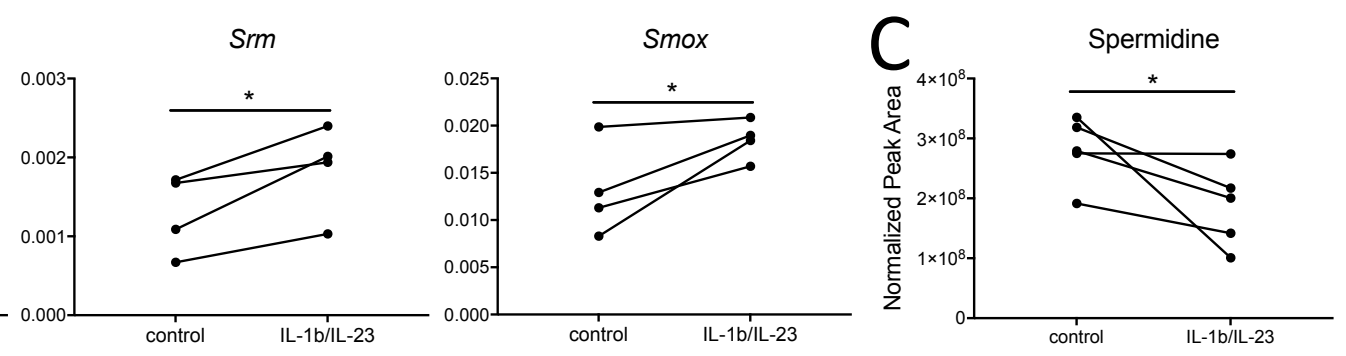

Fig.1 
bioRxiv preprint doi: https://doi.org/10.1101/2021.08.31.458409; this version posted August 31, 2021. The copyright holder for this preprint A (which was not certified by peer review) is the author/funder. All rights reserved. No reuse allowed without permission.
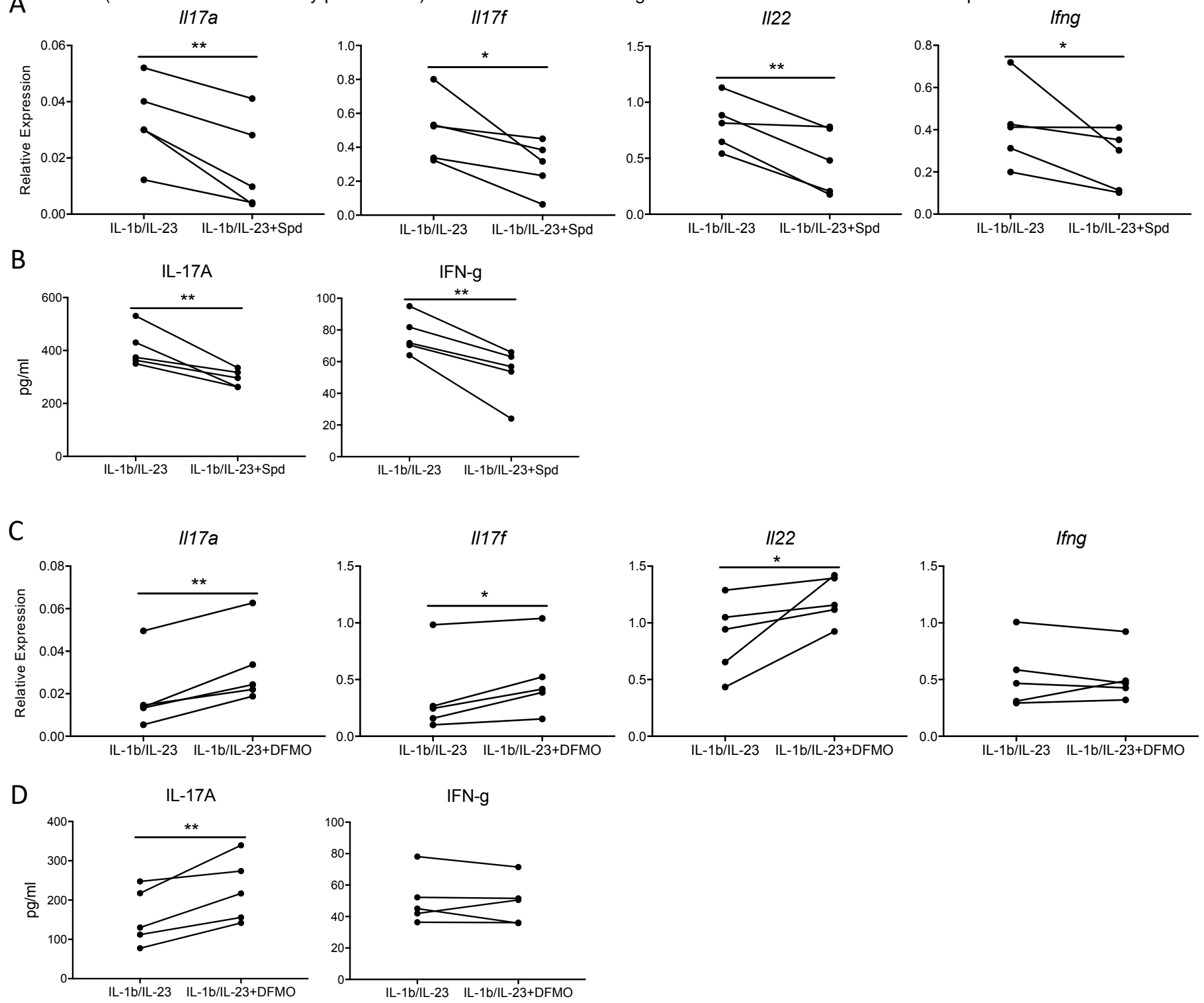

Fig.2 
bioRxiv preprint doi: https://doi.org/10.1101/2021.08.31.458409; this version posted August 31, 2021. The copyright holder for this preprint

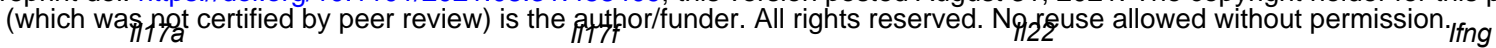
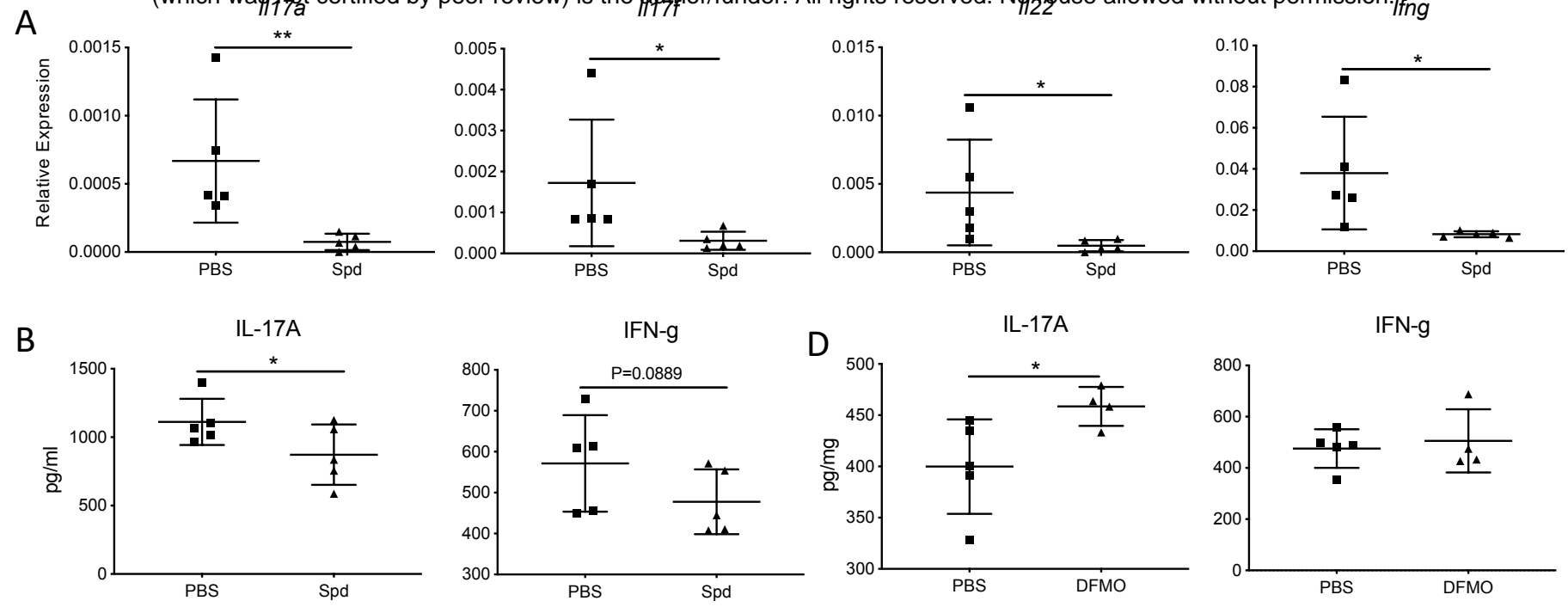

IFN-g
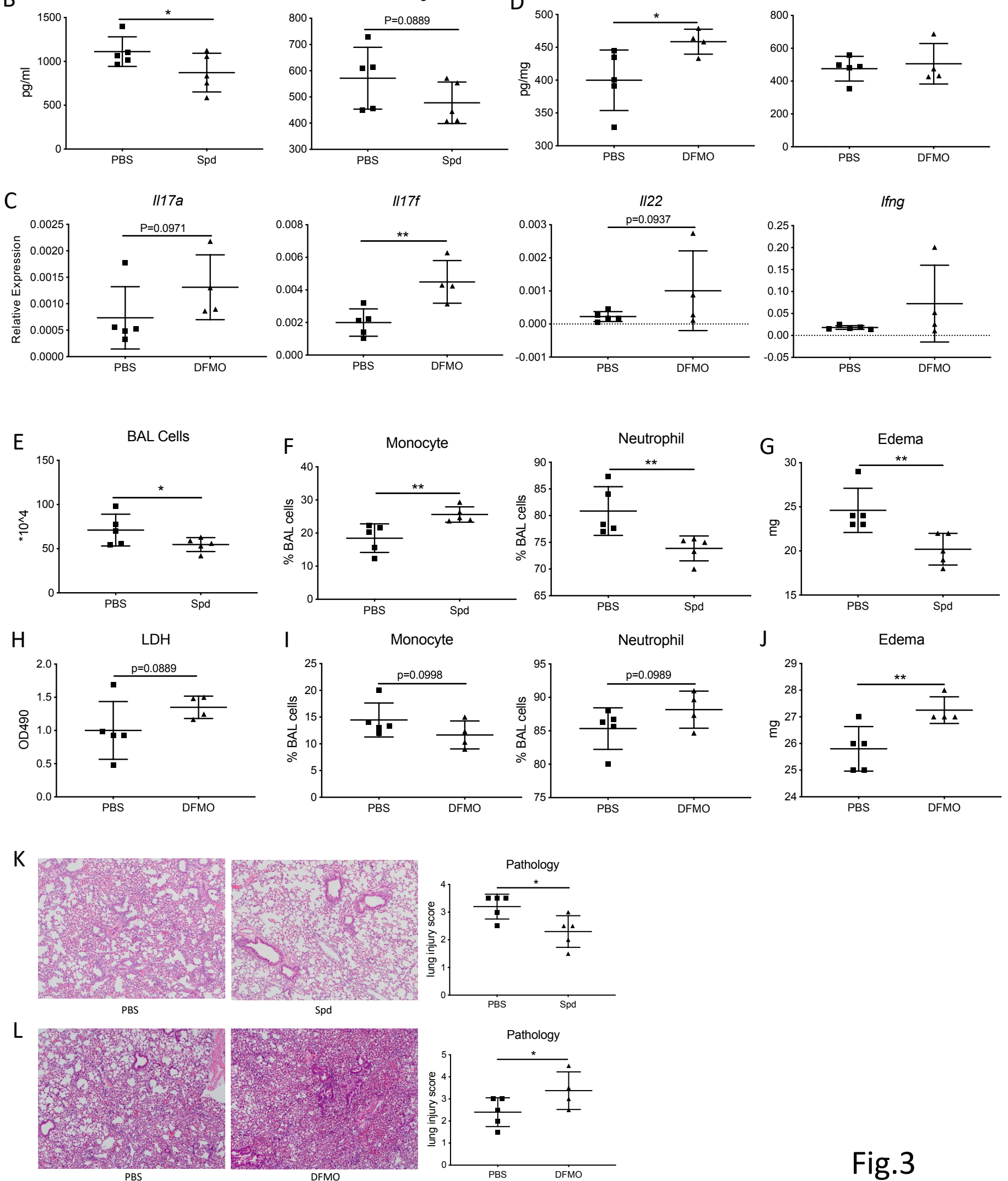

Fig.3 
bioRxiv preprint dol: https://doi.org/10.1101/2021.08.31.458409; this version posted August 31, 2021. The copyright holder for this reprint

A (which was not certified by peer review) is the author/funder. All rights reserved. No reuse allowed without permission.
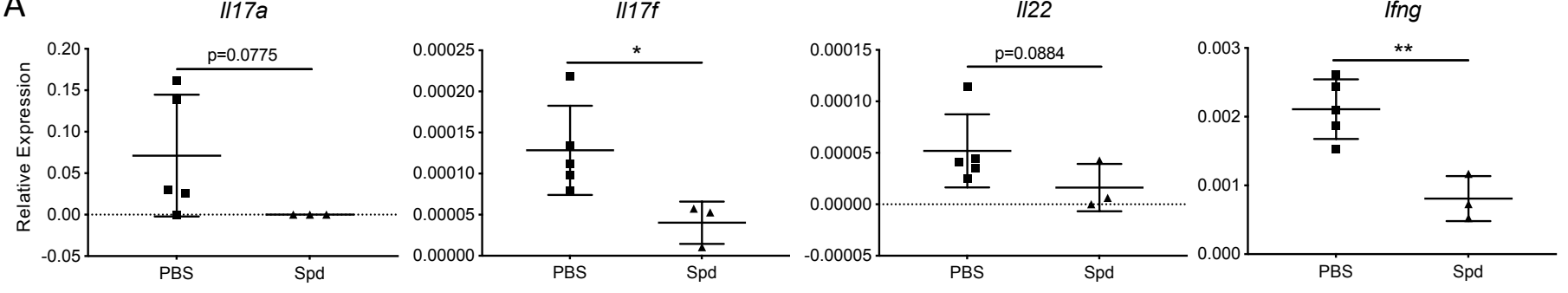

B

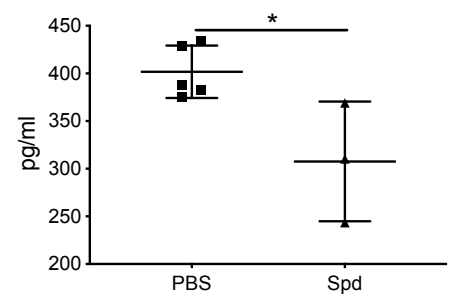

D
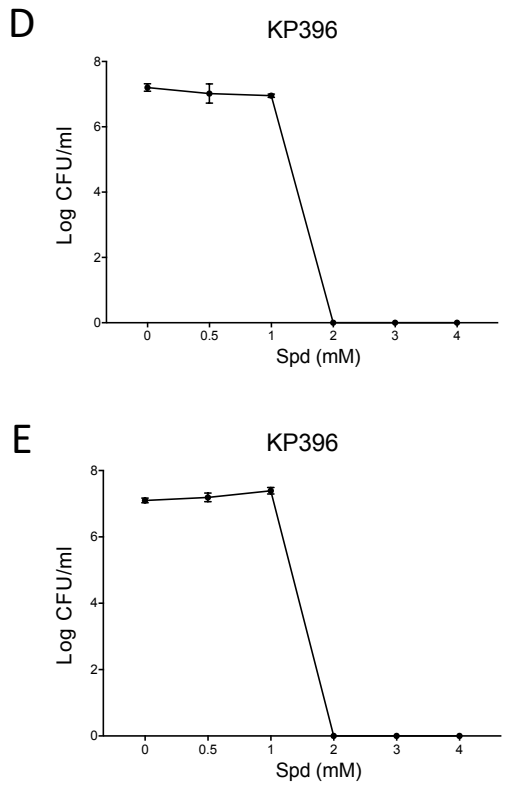

IFN-g

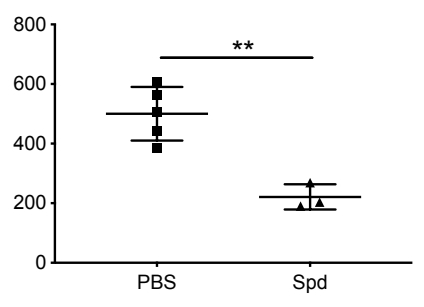

KP-NDM
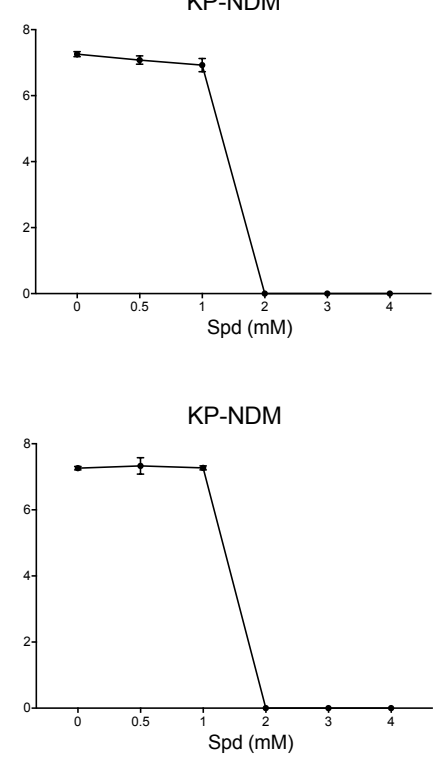

C

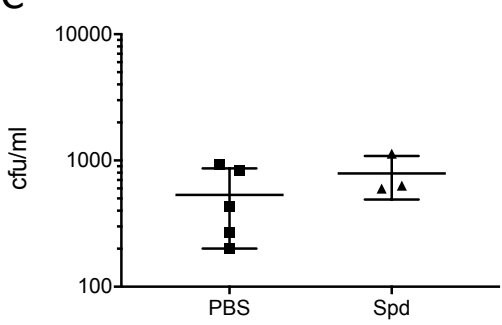

PA

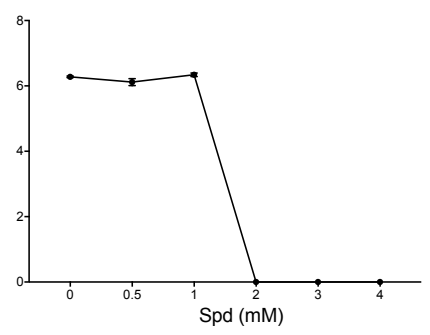

PA

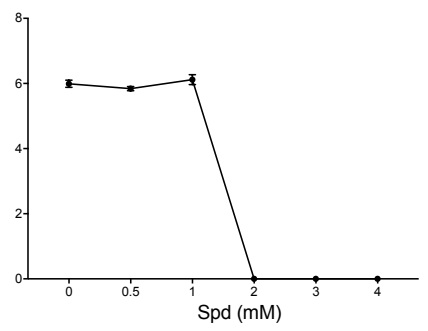

PA14

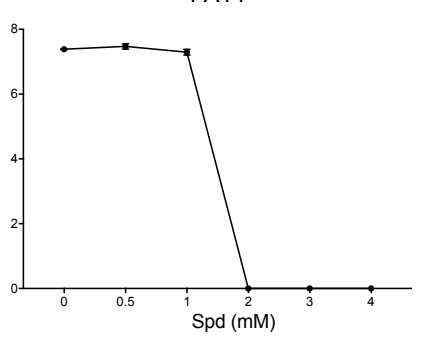

PA14

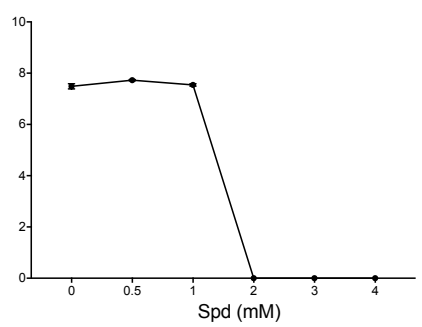

Fig.4 
bioRxiv preprint doi: https://doi.org/10.1101/2021.08.31.458409; this version posted August 31, 2021. The copyright holder for this preprint (which was not certified by peer review) is the author/funder. All rights reserved. No reuse allowed without permission.

A

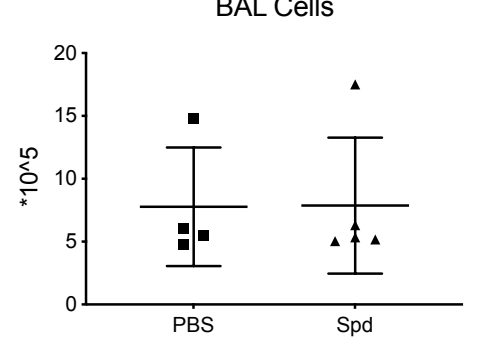

B

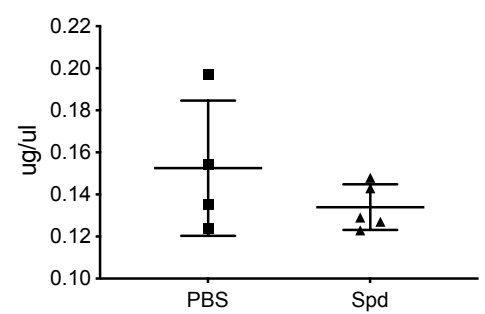

C

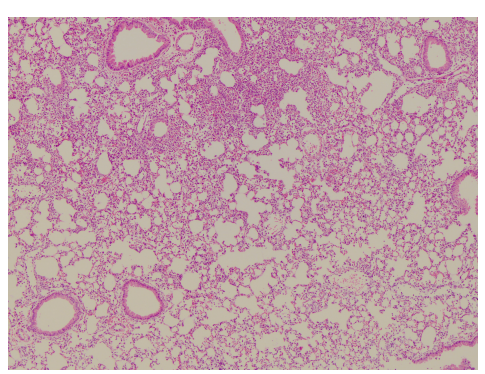

PBS

D

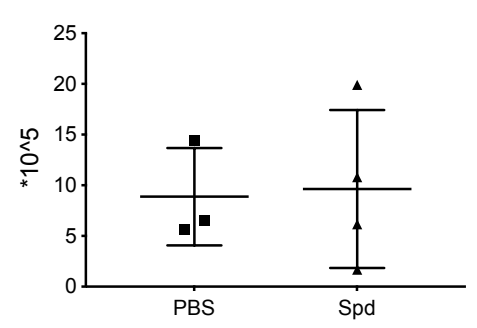

E

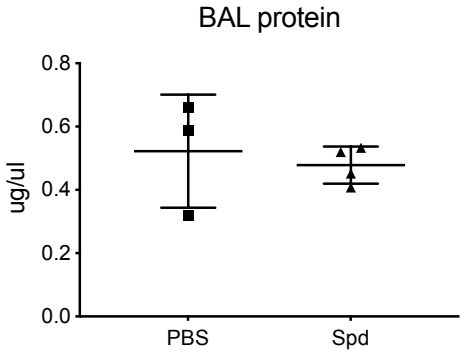

F

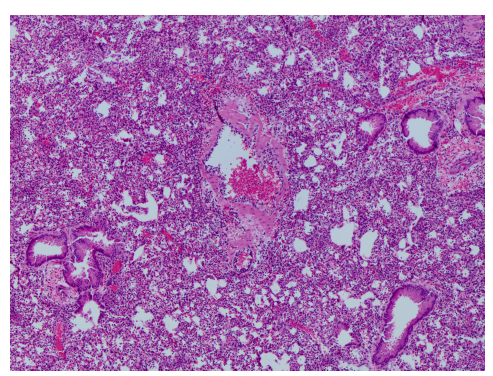

PBS
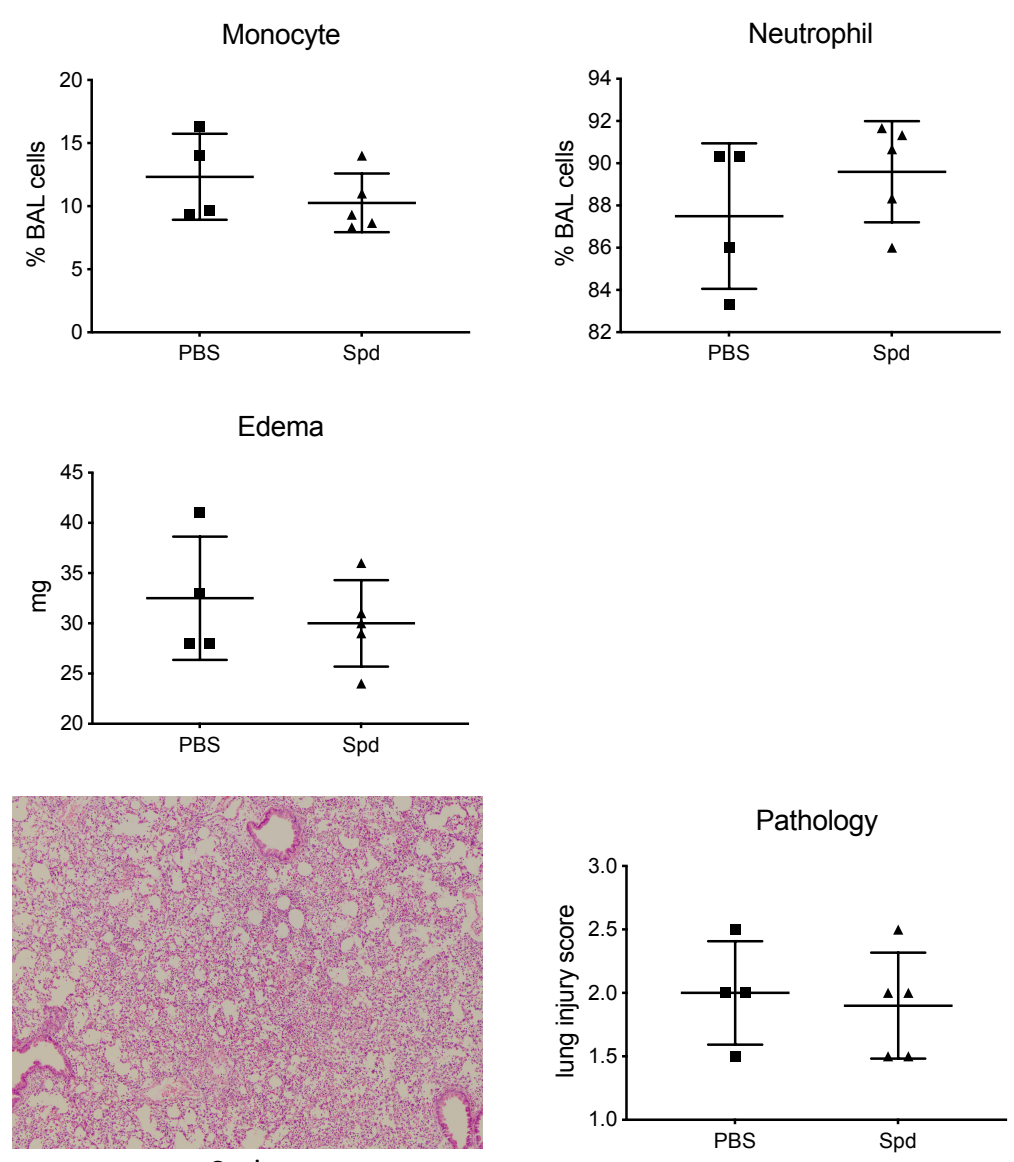

Spd
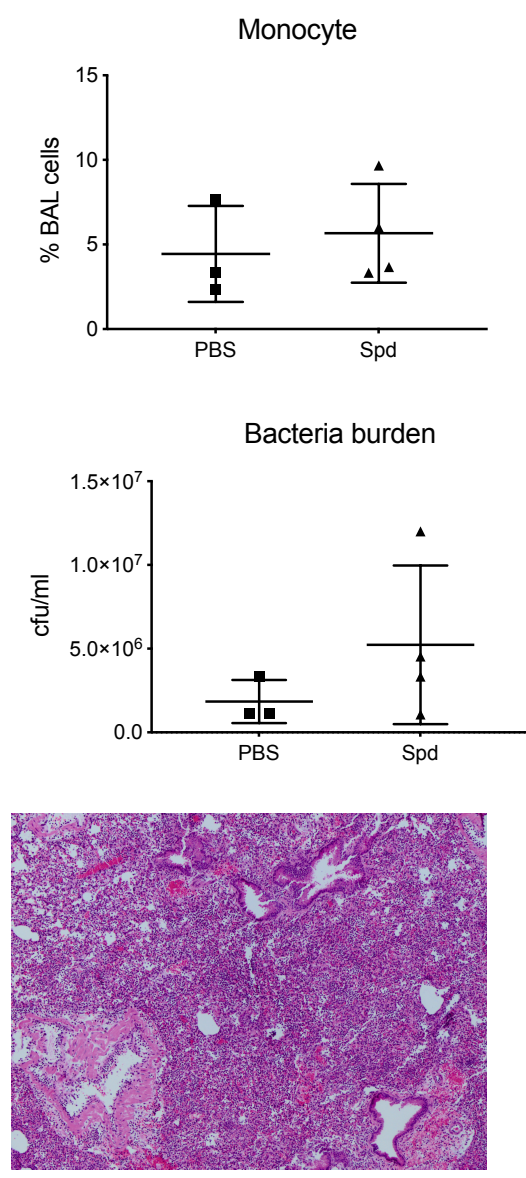

Spd
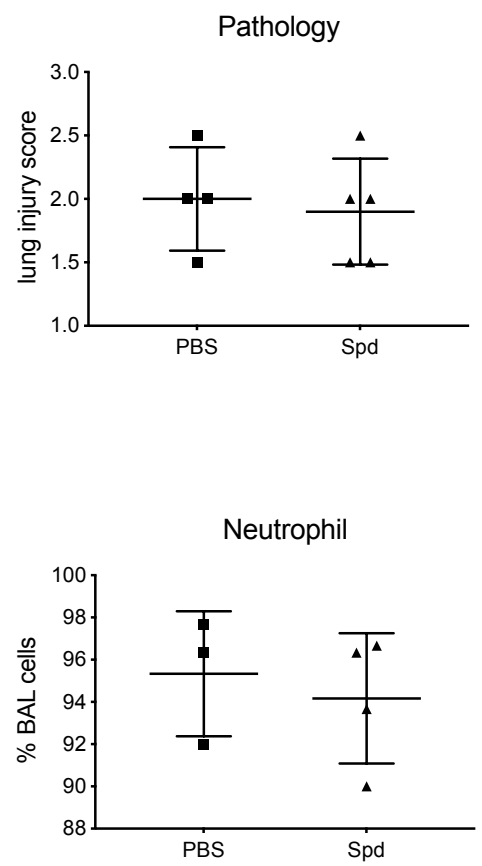

Pathology

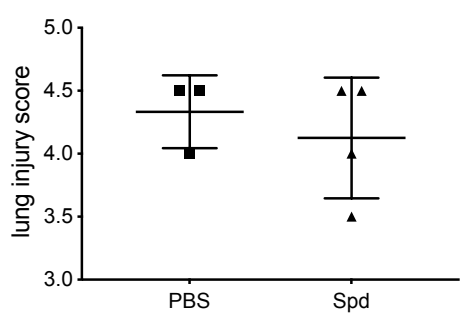

Fig.5 
bioRxiv preprint doi: https://doi.org/10.1101/2021.08.31.458409; this version posted August 31, 2021. The copyright holder for this preprint (which was not certified by peer review) is the author/funder. All rights reserved. No reuse allowed without permission.

A

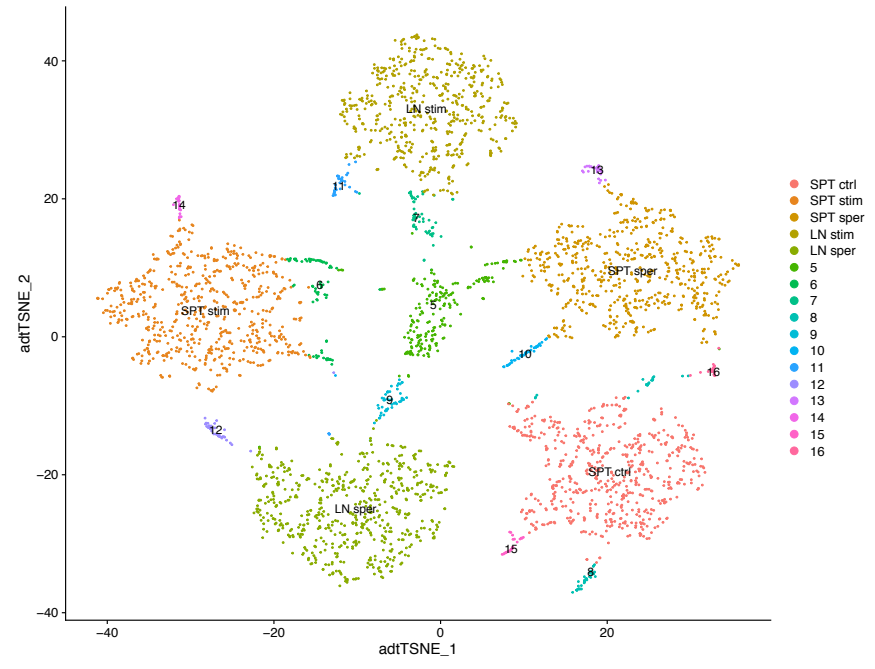

C
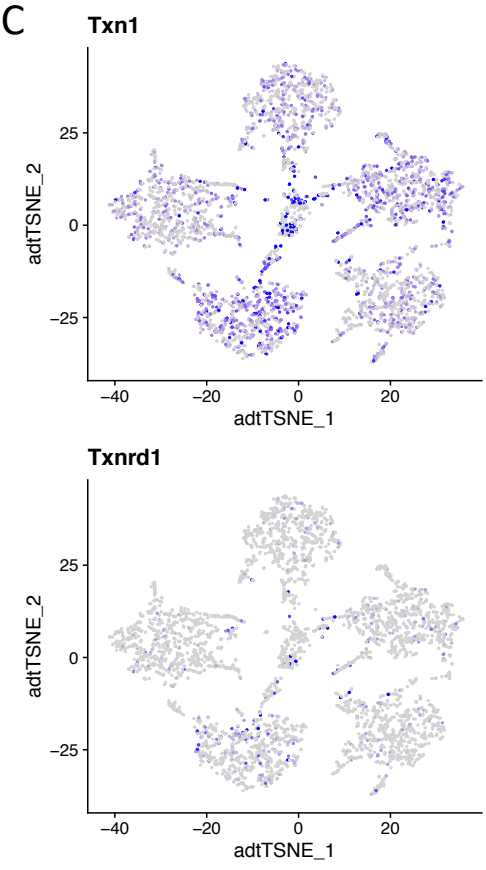

B

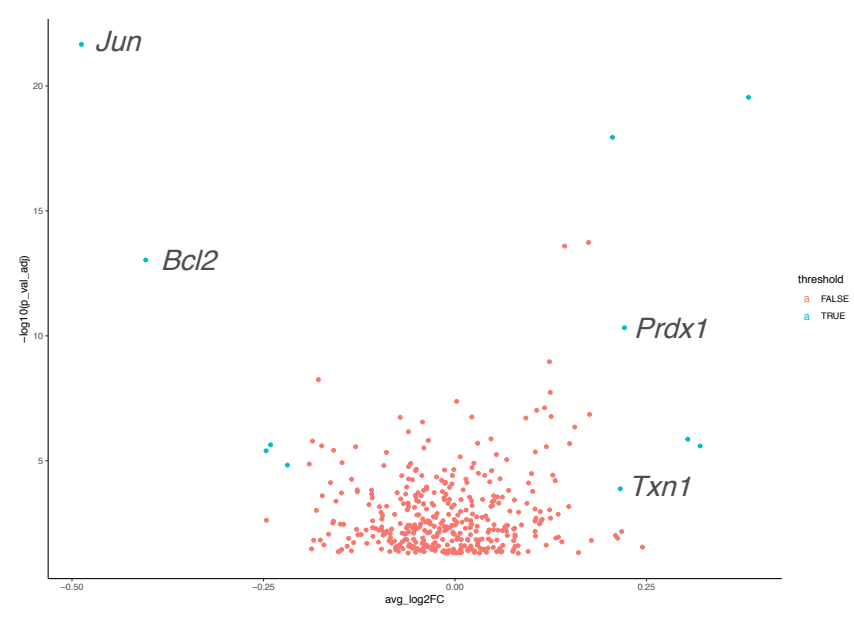

D

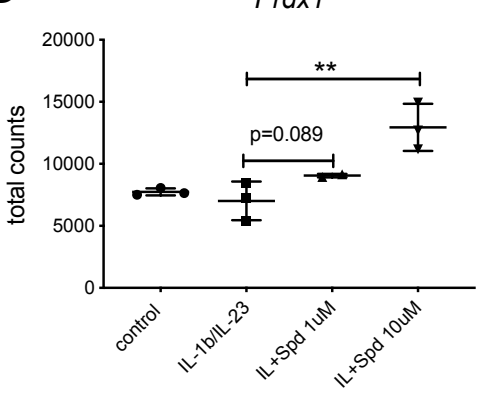

E

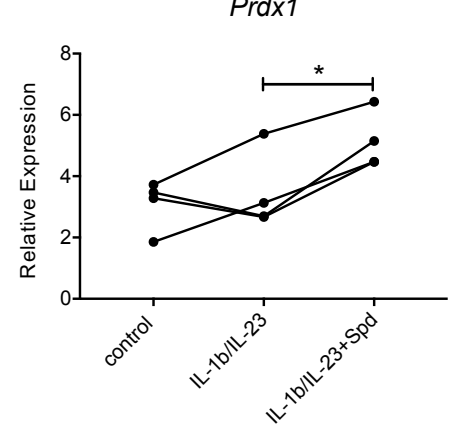

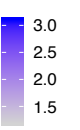

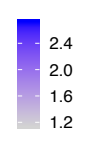

F

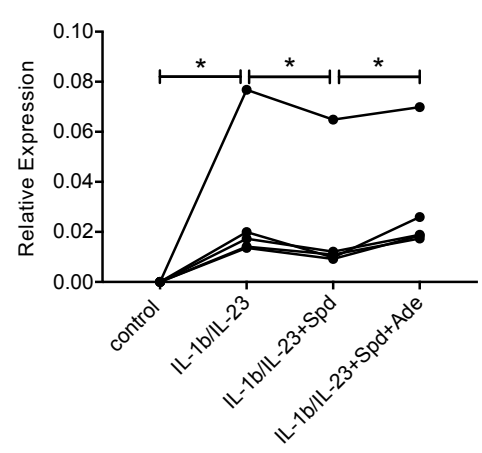

G

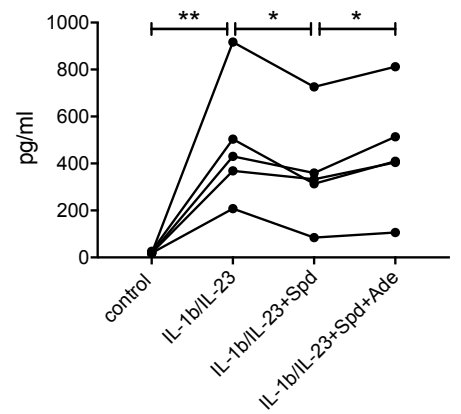

Prdx1

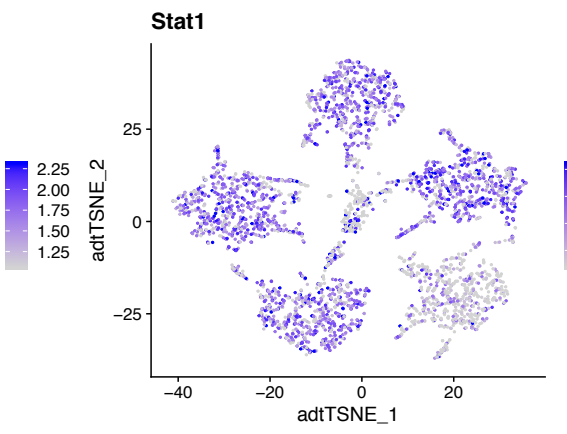

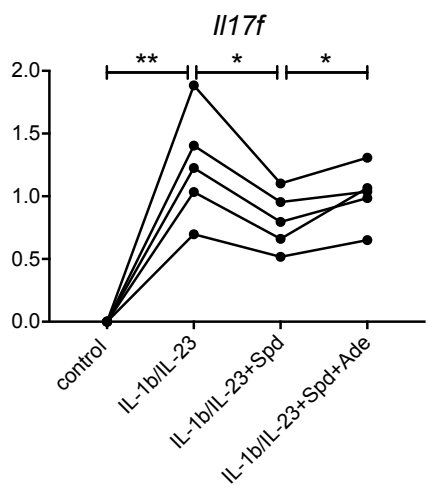

IFN-g

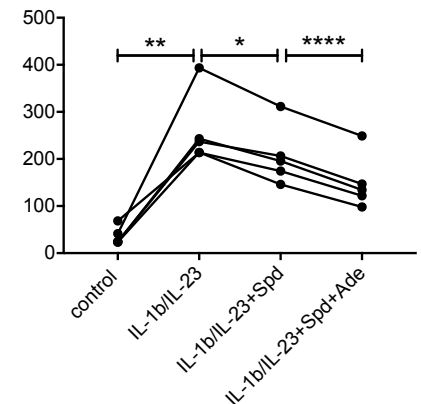

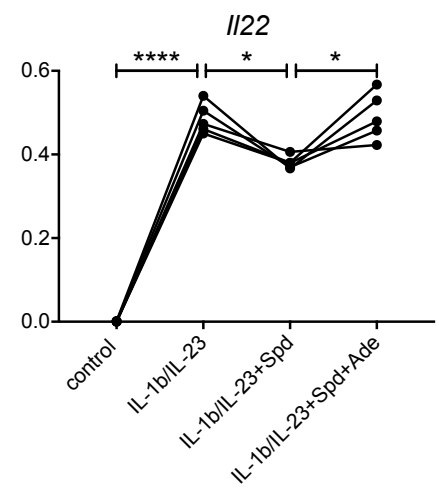

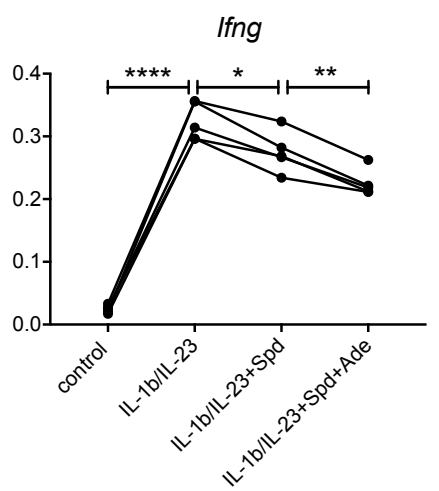

Fig.6 
Figure 1. Polyamine metabolism is involved in IL-17 production.

A: Mouse peritoneal lavage cells induced by thioglycollate were collected and stimulated with IL-1b/IL-23, and scRNA data confirmed IL-17 producing cells have high Srm and Smox expression.

$B$ and C: Fresh enriched spleen T cells were stimulated with mouse IL-1b +IL-23 50ng/ml overnight, (B) I/17a, Srm and Smox were highly induced, detected by qPCR, gene expression relative to Gapdh (graphs displayed in panel B are representative ones from 4 repeated experiments, $\mathrm{N}=2-4$ in each experiment); (C) Polyamine in the cytoplasm of T cells were measured by HPLC system.

Figure 2: Spermidine downregulates the Th17/IL-17 by directly targeting T cells in vitro. Fresh enriched spleen T cells were stimulated with mouse IL-1b $+\mathrm{IL}-2350 \mathrm{ng} / \mathrm{ml}$ at the presence or absence of 3uM Spermidine or DFMO treatment overnight. This is a representative graph of five experiments, $\mathrm{N}=2-4$ in each experiment.

A: $/ / 17 a,\|17 f\| /$,22 and Ifng gene expression was measured by qPCR

B: Relative expression to Hprt; IL-17A and IFN-g proteins were tested by ELISA.

C: $\|17 a\| 17 f,, \| / 22$ and $I f n g$ gene expression was measured by qPCR relative to Hprt expression

D: IL-17A and IFN-g proteins were tested by ELISA

Figure 3. Spermidine dampens inflammation through inhibiting IL-17 production in an acute lung injury (ALI) in vivo model. $\mathrm{N}=5$ in both PBS and Spd group.

A-B: Lung tissue $/ / 17 a$ and Ifng mRNA (C) and protein (D) productions were obtained by ELISA and $\mathrm{qPCR}$, relative expression to Hprt.

C-D: Blockage of spermidine synthesis by DFMO in ALI mice model ( $N=5$ in PBS group; $N=4$ in DFMO group). IL-17 family production at mRNA level (C) and protein level (D) in lung tissue were obtained by ELISA and qPCR, relative expression to Hprt.

E: BAL cells were counted in PBS and spermidine treated lungs from ALI mouse models.

F: BAL cell differentiation including monocyte and neutrophil were identified and quantified under the microscope after Kwik-Diff Staining.

G: Right middle lung lobe was incubated in a $65 \mathrm{C}$ incubator for $48 \mathrm{~h}$, wet/dry weight ratio was recorded and edema levels were then calculated by wet weight minus dry weight

$\mathrm{H}$ : DFMO administration resulted in higher levels of $\mathrm{LDH}$, indicating more inflammation mediated cytotoxicity.

I: DFMO group displayed less Monocytes and more Neutrophils in BAL cells.

J: DFMO group displayed more severe lung edema, as calculated before in Figure 3G.

K: Further lung tissue histology injury score was assessed. Inflammation can be seen in both groups. Compared to the PBS group, Spermidine group displayed less inflammatory cells, slighter alveolar space congestion, and less alveolar wall damage/alveolar fusion. This experiment has been repeated once.

L: Further lung tissue histology injury score was assessed. Compared to the PBS group, DFMO group revealed more inflammatory cells infiltration, stronger alveolar space congestion and higher injury score. This experiment has been repeated once. 
Figure 4: Spermidine treatment suppresses Th17/IL-17 pathway in a live PA bacteria infected mouse model.

A: Spermidine alleviates Th17/IL-17 pathway production in PA infected mice model ( $N=5$ in PBS group; $\mathrm{N}=3$ in Spd group). Th17/IL-17 pathway gene mRNA and protein in lung tissue were achieved by RT-PCR and ELISA, relative expression to Hprt.

B: Lung PA bacteria burden (K) was obtained with cfu assay. This experiment has been repeated once.

C: CFU assay to determine bactericidal activity of varying concentrations of spermidine after 4 hours. Live bacteria including KP396, KP-NDM, PA (as PA ATCC10145) and PA14 were treated with $0,0.5,1,2,3$ and $4 \mathrm{mM}$ spermidine.

D: CFU assay to determine bactericidal activity of varying concentrations of spermidine after 24 hours. Live bacteria including KP396, KP-NDM, PA (as PA ATCC10145) and PA14 were treated with $0,0.5,1,2,3$ and $4 \mathrm{mM}$ spermidine.

Figure 5. Lymphocyte depleted Rag2/II2rg double KO mice do not respond to spermidine. 6-8 weeks Rag2/II2rg double knockout mice were injected with Spermidine $50 \mu \mathrm{g} / \mathrm{g}$ according to mouse weight or PBS by IP twice at $-24 \mathrm{~h}$ and $0 \mathrm{~h}$ separately. Then mice from both groups were inoculated with $5 \mu \mathrm{g} / \mathrm{g}$ LPS (A-C) according to mouse weight or $1^{*} 10^{\wedge} 6 \mathrm{cfu}$ PA14 live bacteria (D-F) by IT at $0 \mathrm{~h}$. $24 \mathrm{~h}$ later, BAL and lung tissue were harvested for downstream analysis. A-C: Immune cell differentiation (A), BAL protein and lung tissue edema (B) histology (C) were obtained in LPS induced ALI model; D-F: In live bacterial infection model, immune cell differentiation (D), BAL protein, bacteria burden (E) and pathology (F) were also assessed.

Figure 6. Spermidine inhibits IL-17 production in a Prdx1 dependent manner.

A: scRNA-Seq integration and cell cluster label of fresh lymph node cells and enriched spleen $T$ cells, which were stimulated with mouse IL-1b $+\mathrm{IL}-2350 \mathrm{ng} / \mathrm{ml}$ at the presence or absence of 3uM Spermidine treatment overnight. Cells were collected for scRNA-Seq performance. 5 major cell population was identified by scATAC-seq data: spleen T cell control group (SPT ctrl); spleen T cell IL-1b/IL-23 stimulation group (SPT stim); spleen T cell IL-1b/IL-23 plus spermidine treatment group (SPT sper); lymph node cell IL-1b/IL-23 stimulation group (LN stim); lymph node cell IL-1b/IL-23 plus spermidine treatment group (LN sper).

B: Antioxidant activity related genes Txn1 and Prdx1 were found significantly upregulated after spermidine treatment to IL-1b/IL23 activated IL-17 producing cells.

C: Volcano plots representing differentially expressed genes between IL-1b/IL-23 stimulation group vs IL-1b/IL-23 plus spermidine treatment group (cutoffs: LogFC=0.2 and padj=0.001).

D-E: Both mRNA-seq data (D) and RT-PCR (E) revealed Prdx1 as a potential target for spermidine, relative to $H$ prt expression, $\mathrm{N}=3$.

F-G: Spermidine inhibits IL-17 production in a $\operatorname{Prdx} 1$ dependent manner. Fresh enriched spleen T cells were stimulated with $50 \mathrm{ng} / \mathrm{ml}$ mouse IL-1b +IL-23, 50ng/ml IL-1b/IL-23 + 3uM Spermidine, 50ng/ml IL-1b/IL-23 + 3uM Spermidine + 10uM Prdx1 inhibitor Adenanthin (Ade) separately, incubating overnight. Ade treatment reversed the suppression of Th17/IL-17 pathway induced by spermidine. $/ / 17 a,\|17 f\|$,22 and Ifng gene expression was measured by qPCR (F), relative expression to Hprt; IL-17A and IFN-g proteins were tested by ELISA (G). This figure is a representative of four experiments, $\mathrm{N}=2-3$ in each experiment. 